\title{
ANTÍGENOS HLA ESTÃO ASSOCIADOS A RISCO DE DIABETES MELLITUS PÓS-TRANSPLANTE?
}

\section{Are human leukocyte antigen complexes associated with risk to post-transplant diabetes mellitus?}

\author{
Débora Dias de Lucena', Renato de Marco², João Roberto de Sá ${ }^{3}$, José Osmar Medina-Pestana1, \\ Maria Gerbase-DeLima², Érika Bevilaqua Rangel ${ }^{1}$
}

\section{RESUMO}

Introdução: Diabetes mellitus pós-transplante (DMPT) tem impacto na morbidade e na mortalidade em receptores de transplante renal, uma vez que aumenta o risco de complicações cardiovasculares e de perda do enxerto renal. Os fatores de risco para DMPT podem ser divididos em modificáveis e não modificáveis. Ainda há controversas sobre a influência do sistema de antígenos leucocitários humanos (Human Leukocyte Antigens ou HLA) no risco de DMPT. Objetivo: Verificar prevalência para DMPT e sua relação com fatores genéticos. Métodos: Foram avaliados 450 pacientes submetidos ao transplante renal e realizada a investigação quanto à associação entre antígenos HLA-A, -B e -DR e DMPT. As frequências dos antígenos HLA-A, -B e -DR foram comparadas entre receptores com e sem diagnóstico de DMPT, ao longo de três anos após o transplante. Resultados: Na população de estudo, $60 \%$ eram do sexo masculino, $47,2 \%$ eram negros e $57,8 \%$ receberam rim de doador falecido. DMPT foi diagnosticado em 61 pacientes (13,5\%), 315 (70\%) permaneceram com níveis glicêmicos normais e 74 (16,5\%) desenvolveram glicemia de jejum alterada. Associações positivas de DMPT foram observadas em relação aos antígenos HLA-A23 (18\% vs $8,7 \%, P=0,024)$ e HLA-A29 $(13,1 \%$ vs $5,1 \%, P=0,017)$ e associação negativa com o antígeno HLA-A68 $(4,9 \%$ vs $14,1 \%, P=0,046)$. Conclusão: Encontramos relação estatística entre DMPT e HLA- A23, HLA-A29 e HLA-A68, porém, estudos com maior número de indivíduos são necessários para comprovar definitivamente a associação entre antígenos HLA e o risco de DMPT.

Keywords: Diabetes mellitus; Transplante de Rim; Fatores de Risco; Antígenos HLA.

\footnotetext{
Instituições:

${ }^{1}$ Disciplina de Nefrologia, Universidade Federal de São Paulo (UNIFESP)/ Hospital do Rim e Hipertensão, São Paulo/SP.

2 Instituto de Imunogenética (IGEN), Associação Fundo de Incentivo à Pesquisa, São Paulo/SP.

${ }^{3}$ Disciplina de Endocrinologia, Universidade Federal de São Paulo (UNIFESP), São Paulo/SP.
}

\section{Correspondência:}

Débora Dias de Lucena

E-mail: deblucena@yahoo.com.br

https://doi.org/10.53855/bjt.v24i1.001

Recebido em: 28/12/2020
Aceito em: $17 / 03 / 2021$

\section{INTRODUÇÃO}

O diabetes mellitus pós-transplante (DMPT) contribui para o aumento da morbidade e mortalidade de causa cardiovascular em receptores de transplante renal, com prevalência relatada em 10 a $20 \%$ dessa população. ${ }^{1,2}$

Os fatores de risco para DMPT podem ser divididos em modificáveis e não modificáveis. Os modificáveis incluem: atividade física, índice de massa corpórea (IMC), síndrome metabólica, hiperglicemia prétransplante, relação entre triglicerídeos e colesterol HDL ( $\mathrm{Tg} / \mathrm{HDL} \geq 3,5)$, infecção pelo vírus da hepatite $\mathrm{C}$ e pelo citomegalovírus (CMV), uso de imunossupressores, nível sérico de tacrolimo, rejeição aguda e proteinúria. ${ }^{3-5}$ Os fatores de risco não modificáveis incluem idade, história familiar de DM, doença renal policística autossômica 
dominante, etnia negra, algumas populações hispânicas e maior número de incompatibilidades no sistema de antígenos leucocitários humanos ou human leukocyte antigens (HLA). ${ }^{3-5}$

Estudos sobre associação entre antígenos HLA e susceptibilidade a DMPT, apesar de já terem sido realizados em várias populações, não revelaram, até o momento, resultados consistentes. ${ }^{6-11}$

A recomendação inicial na abordagem da intolerância à glicose e DMPT é baseada na identificação precoce de pacientes com risco de desenvolver hiperglicemia pós-transplante, bem como na atuação sobre os fatores de risco modificáveis com mudanças de estilo de vida, seguida de rápida intervenção terapêutica medicamentosa. ${ }^{12-14}$

O presente estudo teve como objetivo verificar a prevalência de DMPT e sua relação com fatores genéticos representados por antígenos HLA numa coorte de pacientes transplantados renais, avaliados ao longo dos três primeiros anos, em um centro único.

\section{MÉTODOS}

Foi feito um estudo de coorte, transversal, observacional e descritivo, após submissão e aprovação pelo Comitê de Ética em Pesquisa da Universidade Federal de São Paulo-Escola Paulista de Medicina (UNIFESP-EPM) (protocolo: 66288217.0.0000.5505). Foram incluídos 450 pacientes transplantados renais de doadores falecidos ou vivos, no Hospital do Rim e Hipertensão de São Paulo, durante o ano de 2011. Nesse ano, foram realizados 932 transplantes na instituição, de modo que obtivemos o número de 450 pacientes, a partir de cálculo amostral, sendo os prontuários revisados de modo sequencial. Todos os indivíduos foram acompanhados por pelo menos três anos, exceto aqueles que foram a óbito $(n=21)$ ou que evoluíram com perda do enxerto $(n=1)$ antes do final do estudo. Excluímos 104 pacientes que tinham diagnóstico de diabetes mellitus (DM) antes do transplante, 49 submetidos ao transplante simultâneo de pâncreas-rim, 10 submetidos ao transplante de pâncreas após transplante de rim, 41 menores de 18 anos, 239 que foram transferidos para outro hospital e quatro retransplantes renais.

Todos os pacientes receberam metilprednisolona $1,0 \mathrm{~g}$ durante a indução, no período intraoperatório. Para os pacientes com PRA $\geq 50 \%$, foi realizada indução com timoglobulina $1 \mathrm{mg} / \mathrm{kg} / \mathrm{dia}$ (variando de três a seis doses). A imunossupressão de manutenção foi baseada no uso de Tacrolimo (FK), prednisona (PRED) e micofenolato de sódio (MPS) em pacientes com Reatividade de Anticorpo contra Painel (PRA) $\geq 50 \%$ ou PRA $<50 \%$ com doadores de critério expandido. Para PRA $<50 \%$ com doadores padrão, foram utilizados esquemas de FK, PRED e azatioprina (AZA) ou FK, PRED e inibidores da mammalian target of rapamycin (imTOR). Para receptores de doadores vivos com baixo risco imunológico (haplo-idênticos, PRA $<50 \%$ e primeiro transplante), o regime imunossupressor foi baseado em ciclosporina (CsA), PRED e AZA. O período da pesquisa coincidiu com o início dos testes de alguns protocolos, por isso alguns pacientes incluíam no seu esquema de imunossupressão sirolimo ou everolimo.

A dose cumulativa de corticosteróides foi calculada através da dose de prednisona, em miligramas $(\mathrm{mg})$, nos primeiros seis meses após o transplante, incluindo também a dose de metilprednisolona no período perioperatório (1g) e aquela dose utilizada como pulsoterapia para o tratamento de rejeição aguda do enxerto renal (1g/dia, 3-5 doses, dependendo do grau da rejeição aguda) e, em seguida, ajustada pelo peso corporal em quilogramas $(\mathrm{kg})$.

A hiperglicemia transitória foi definida por duas medições de glicemia de jejum alterada $\geq 126 \mathrm{mg} / \mathrm{dl}$ ou glicose plasmática aleatória $\geq 200 \mathrm{mg} / \mathrm{dl}$, dentro de três meses após o transplante renal. Quando esses parâmetros foram observados, após três meses (12 semanas) do transplante renal, definimos como DMPT. Glicemia de jejum alterada foi definida como duas medidas de glicemia de jejum medidas entre $110-125 \mathrm{mg} / \mathrm{dl}$.

Para investigação da associação entre antígenos HLA-A, -B e -DR e DMPT, utilizamos a tipificação HLA dos receptores, realizada por PCR (Polymerase Chain Reaction)-SSP (Sequence Specific Primers) ou PCRSSO (Sequence Specific Oligonucleotides), por ocasião da inclusão do paciente na lista de espera de transplante renal. ${ }^{15}$ As frequências dos antígenos HLA-A, -B e -DR foram comparadas entre receptores com e sem DMPT. As frequências desses antígenos HLA na população geral, representada pela população de doadores, foi fornecida pelo Instituto de Imunogenética, AFIP e é apresentada nos resultados. O teste do qui-quadrado de Pearson foi realizado para as variáveis categóricas ou nominais, enquanto para variáveis numéricas utilizamos o t-test. $P<0,05$ foi considerado estatisticamente significante. Utilizamos o programa Statistical Productand Services Solutions, versão 18.0, SPSS Inc, Chicago, IL, EUA e GraphPad Prisma (versão 7.0, San Diego, CA. EUA).

\section{RESULTADOS}

$\mathrm{Na}$ tabela 1, descrevemos as características demográficas dos 450 pacientes transplantados incluídos no estudo. DMPT foi diagnosticado em 61 
pacientes $(13,5 \%)$, enquanto $315(70 \%)$ permaneceram com níveis glicêmicos normais e $74 \quad(16,5 \%)$ desenvolveram glicemia de jejum alterada.

Tabela 1 -Características dos pacientes

\begin{tabular}{lccc}
\hline \multicolumn{1}{c}{ Variáveis } & & $\mathbf{N}$ & $\%$ \\
\hline \multirow{2}{*}{ Sexo } & Feminino & 180 & 40 \\
& Masculino & 270 & 60 \\
Faixa etária & $18-39$ anos & 186 & 41,3 \\
& $40-59$ anos & 240 & 53,3 \\
Média de idade & $\geq 59$ anos & 24 & 5,3 \\
& 45 anos & & \\
IMC (kg/m2) & $<18$ & 33 & 7,3 \\
& $18-24,9$ & 274 & 60,8 \\
& $25-29,9$ & 109 & 24,2 \\
Cor & $\geq 30$ & 34 & 7,5 \\
& Branco & 238 & 52,8 \\
DRP & Negro/pardo & 212 & 47,2 \\
& Sim & 42 & 9,4 \\
TRS & Não & 408 & 90,6 \\
& Sim & 417 & 92,6 \\
Tipo de doador & Não & 33 & 7,4 \\
& Vivo & 190 & 42,2 \\
& Falecido & 260 & 57,8 \\
\hline
\end{tabular}

IMC: índice de massa corporal, DRP: doença renal policística, TRS: terapia renal substitutiva.

Na tabela 2, mostramos a comparação das frequências dos antígenos HLA-A, -B e -DR entre receptores com e sem DMPT. Frequências significantemente mais elevadas, ou seja, associações positivas, foram observadas em relação a HLA-A23 (18\% vs $8,7 \%$, $\mathrm{P}=0,024)$ e HLA-A29 $(13,1 \%$ vs $5,1 \%, \mathrm{P}=0,017)$. Por outro lado, observamos frequência diminuída (associação negativa) com HLA-A68 (4,9\% vs $14,1 \%, P=0,046)$.

Entretanto, essas associações não podem ser consideradas definitivas, porque $O$ valor de $P$ perde a significância quando é corrigido para múltiplas comparações, isto é, multiplicado pelo número de antígenos analisados.

$\mathrm{Na}$ tabela 2 também estão apresentadas as frequências dos antígenos HLA na população geral, representada pela população de doadores de órgãos, mas essas frequências não foram consideradas nas análises estatísticas.

Também foram encontrados outros fatores que puderam predizer o risco de DMPT, como: hiperglicemia prétransplante $(P=0,032)$, IMC pré-transplante $\geq 25 \mathrm{~kg} / \mathrm{m} 2$ $(P=0,0001)$, ocorrência de rejeição aguda do enxerto renal $(P=0,021)$, hiperglicemia transitória $(P=0,0001)$, idade do receptor $(P=0,0001)$, relação $T G / H D L \geq 3,5$ $(P=0,0001)$, bem como nível sérico de FK $(P=0,004) .^{5}$

\section{DISCUSSÃO}

Verificamos prevalência de $13,5 \%$ de DMPT, seguindo a tendência de outros estudos que descreveram prevalências de $7,4 \%$ a $32 \% .{ }^{4,16}$

A fim de identificarfatores genéticos de risco envolvidos no DMPT, avaliamos associação entre antígenos HLA-A, B e DR e DMPT. Observamos frequências significantemente elevadas de HLA-A23 e HLA-A29 (associações positivas ou de susceptibilidade) e diminuída de HLA-A68 (associação negativa ou de resistência) em receptores com DMPT, comparados aos sem DMPT. Entretanto, essas associações não podem ser consideradas definitivas, porque o valor de $P$ perde a significância quando corrigido para as múltiplas comparações realizadas. Dessa forma, são necessários novos estudos sobre a possível influência de genes HLA na susceptibilidade e resistência a DMPT em nossa população. A falta de homogeneidade de cor/raça entre os receptores analisados em nosso estudo pode ser considerada um fator limitante, porém não parece ter influenciado os resultados, uma vez que, neste trabalho, cor não foi fator preditor de DMPT.

É importante ressaltar que nenhuma das associações encontradas em nosso trabalho foram relatadas previamente em nenhuma das várias populações estudadas por outros autores e que também não há uniformidade de achados entre os trabalhos publicados. ${ }^{6-11}$ Estes fatos vão contra a hipótese de haver associação entre genes HLA e susceptibilidade ou resistência ao desenvolvimento de DMPT. Por outro lado, considerando os achados do presente trabalho, assim como os trabalhos da literatura que analisaram antígenos HLA-DR, é possível afirmar que os genes HLA-DR que estão associados à susceptibilidade ao diabetes mellitus (HLA-DR3 e HLA-DR4) não se associam com DMPT. ${ }^{7,10}$

Nessa mesma população, identificamos como fatores de risco modificáveis: hiperglicemia pré-transplante, IMC $\geq 25 \mathrm{~kg} / \mathrm{m} 2$, rejeição aguda e hiperglicemia transitória. Não encontramos associação entre gênero e DMPT, ${ }^{5}$ assim como outros estudos que também não demonstraram relação entre gênero e DMPT ou síndrome metabólica pós-transplante renal. ${ }^{3,17} \mathrm{~A}$ cor também não foi fator preditor de DMPT, ${ }^{5}$ apesar de já ter sido demonstrada a relação positiva entre DMPT e etnia afro-americana, embora não relatada diferença entre populações hispânicas e caucasianas para o desenvolvimento de DMPT. ${ }^{3,18}$ No Brasil, por ser um país formado em sua maioria por população miscigenada, há dificuldade para definição de etnias, e por este motivo, não foi possível estabelecer relação entre cor e DMPT. 
Antígenos HLA estão associados a risco de diabetes mellitus pós-transplante?

Tabela 2. Frequência de antígenos HLA em pacientes com e sem DMPT e em população geral, representada por doadores de órgãos tipificados no IGEN-AFIP

\begin{tabular}{|c|c|c|c|c|}
\hline Antígeno HLA & DMPT (+) $\quad(n=61)$ & DMPT (-) $\quad(n=389)$ & $\begin{array}{c}\text { P } \\
\text { DMPT(+) vs DMPT(-) }\end{array}$ & $\begin{array}{c}\text { População geral Doadores) } \\
\text { n=4100 ( \%) }\end{array}$ \\
\hline HLA-A1 & $14,75 \%(9)$ & $14,65 \%(57)$ & 0,983 & $16,6 \%(680)$ \\
\hline HLA-A2 & $57,37 \%(35)$ & $50,64 \%$ (197) & 0,310 & $42,46 \%(1.740)$ \\
\hline HLA-A3 & $13,11 \%(8)$ & $17,99 \%(70)$ & 0,349 & $18,24 \%(748)$ \\
\hline HLA-A11 & $9,83 \%(6)$ & $14,13 \%(55)$ & 0,361 & $10,41 \%$ (427) \\
\hline HLA-A23 & $18 \%(11)$ & $8,7 \%(34)$ & 0,024 & $11,22 \%(460)$ \\
\hline HLA-A24 & $9,83 \%(6)$ & $17,73 \%(69)$ & 0,124 & $16,17 \%(663)$ \\
\hline HLA-A25 & $3,27 \%(2)$ & $2 \%(8)$ & 0,547 & $2,29 \%(94)$ \\
\hline HLA-A26 & $1,63 \%(1)$ & $5,39 \%(21)$ & 0,206 & $5,98 \%(245)$ \\
\hline HLA-A29 & $13,11 \%(8)$ & $5,14 \%(20)$ & 0,017 & 8,39\% (344) \\
\hline HLA-A30 & $11,47 \%(7)$ & $12,85 \%(50)$ & 0,764 & $12,63 \%$ (518) \\
\hline HLA-A31 & $14,75 \%(9)$ & $7,45 \%(29)$ & 0,057 & $8,34 \%(342)$ \\
\hline HLA-A32 & $1,63 \%(1)$ & $4,62 \%(18)$ & 0,281 & $6,02 \%(248)$ \\
\hline HLA-A33 & $4,91 \%$ (3) & $5,91 \%(23)$ & 0,757 & $6,56 \%(260)$ \\
\hline HLA-A34 & $3,27 \%(2)$ & $2 \%(8)$ & 0,547 & $1,80 \%(74)$ \\
\hline HLA-A36 & $0 \%(0)$ & $1,02 \%(4)$ & 0,426 & $1,63 \%(67)$ \\
\hline HLA-A66 & $3,27 \%(2)$ & $1,54 \%(6)$ & 0,340 & $2,27 \%(91)$ \\
\hline HLA-A68 & $4,91 \%(3)$ & $14,13 \%(55)$ & 0,046 & $12,71 \%(521)$ \\
\hline HLA-A74 & $6,55 \%(4)$ & $3,08 \%(12)$ & 0,173 & $3,73 \%(153)$ \\
\hline HLA-A80 & $1,63 \%(1)$ & $0,25 \%(1)$ & 0,131 & $0,51 \%(21)$ \\
\hline HLA-B7 & $8,2 \%(5)$ & $12 \%(47)$ & 0,377 & $14,05 \%(576)$ \\
\hline HLA-B8 & $9,83 \%(6)$ & $10 \%(39)$ & 0,963 & $8,37 \%$ (343) \\
\hline HLA-B13 & $4,91 \%(3)$ & $5,14 \%(20)$ & 0,941 & $3,61 \%(148)$ \\
\hline HLA-B14 & $6,55 \%(4)$ & $12 \%(47)$ & 0,206 & $9,71 \%(398)$ \\
\hline HLA-B15 & $13,11 \%(8)$ & $16,2 \%(63)$ & 0,539 & $19,34 \%$ (793) \\
\hline HLA-B18 & $16,39 \%(10)$ & $9,25 \%(36)$ & 0,087 & $8,54 \%(350)$ \\
\hline HLA-B27 & $8,2 \%(5)$ & $3,85 \%(15)$ & 0,126 & $4,02 \%(165)$ \\
\hline HLA-B35 & $2,82 \%(11)$ & $24,67 \%(96)$ & 0,257 & $20,93 \%$ (858) \\
\hline HLA-B37 & $0 \%(0)$ & $2 \%(8)$ & 0,258 & $2,37 \%$ (97) \\
\hline HLA-B38 & $3,27 \%(2)$ & $4,1 \%(16)$ & 0,757 & $3,12 \%(128)$ \\
\hline HLA-B39 & $6,55 \%(4)$ & $5,14 \%(20)$ & 0,647 & $6,24 \%(256)$ \\
\hline HLA-B40 & $8,2 \%(5)$ & $7,96 \%$ (31) & 0,951 & $9,29 \%$ (381) \\
\hline HLA-B41 & $0 \%(0)$ & $1,54 \%(6)$ & 0,329 & $2,2 \%(90)$ \\
\hline
\end{tabular}




\begin{tabular}{|c|c|c|c|c|}
\hline Antígeno HLA & DMPT $(+) \quad(n=61)$ & DMPT (-) $(n=389)$ & $\begin{array}{c}\text { P } \\
\text { DMPT(+) vs DMPT(-) }\end{array}$ & $\begin{array}{l}\text { População geral Doadores) } \\
\qquad n=4100 \text { ( \%) }\end{array}$ \\
\hline HLA-B42 & $0 \%(0)$ & $5,65 \%(22)$ & 0,057 & $4,22 \%(173)$ \\
\hline HLA-B44 & $22,95 \%$ (14) & $17,22 \%(67)$ & 0,279 & $19,66 \%$ (806) \\
\hline HLA-B45 & $3,27 \%(2)$ & $3,08 \%(12)$ & 0,935 & 4,73\% (194) \\
\hline HLA-B47 & $1,63 \%(1)$ & $0,5 \%(2)$ & 0,315 & 0,37\% (15) \\
\hline HLA-B48 & $1,63 \%(1)$ & $2 \%(8)$ & 0,829 & $1,22 \%(50)$ \\
\hline HLA-B49 & $3,27 \%(2)$ & $5,65 \%(22)$ & 0,442 & 4,78\% (196) \\
\hline HLA-B50 & $3,27 \%(2)$ & $4,37 \%(17)$ & 0,693 & $3,78 \%(155)$ \\
\hline HLA-B51 & $26,22 \%(16)$ & $16,96 \%(66)$ & 0,081 & $14,27 \%(585)$ \\
\hline HLA-B52 & $6,55 \%(4)$ & $4,37 \%$ (17) & 0,451 & $4 \%(164)$ \\
\hline HLA-B53 & $6,55 \%(4)$ & $5,91 \%(23)$ & 0,844 & $5,76 \%(236)$ \\
\hline HLA-B54 & $0 \%(0)$ & $0,5 \%(2)$ & 0,575 & $0,22 \%(9)$ \\
\hline HLA-B55 & $1,63 \%(1)$ & $1,54 \%(6)$ & 0,955 & $2,56 \%(105)$ \\
\hline HLA-B56 & $0 \%(0)$ & $1,79 \%(7)$ & 0,291 & $0,68 \%(28)$ \\
\hline HLA-B57 & $6,55 \%(4)$ & $3,08 \%(12)$ & 0,173 & $5,63 \%(231)$ \\
\hline HLA-B58 & $6,55 \%(4)$ & $4,62 \%(18)$ & 0,516 & $5,69 \%(270)$ \\
\hline HLA-B67 & $0 \%(0)$ & $0,25 \%(1)$ & 0,692 & $0,15 \%(6)$ \\
\hline HLA-B73 & $0 \%(0)$ & $0,25 \%(1)$ & 0,692 & $0,12 \%(5)$ \\
\hline HLA-B78 & $0 \%(0)$ & $0,25 \%(1)$ & 0,692 & $0,15 \%(6)$ \\
\hline HLA-B81 & $1,63 \%(1)$ & $1,02 \%(4)$ & 0,672 & $1,56 \%(64)$ \\
\hline HLA-B82 & $1,63 \%(1)$ & $0,5 \%(2)$ & 0,315 & $0,10 \%(4)$ \\
\hline HLA-DR1 & $14,75 \%$ (9) & $18,25 \%(71)$ & 0,592 & $19,1 \%(784)$ \\
\hline HLA-DR3 & $11,47 \%(7)$ & $20 \%(78)$ & 0,112 & $17,9 \%(732)$ \\
\hline HLA-DR4 & $27,86 \%(17)$ & $21,33 \%(83)$ & 0,297 & $23,5 \%(963)$ \\
\hline HLA-DR7 & $29,5 \%(18)$ & $22,87 \%(89)$ & 0,258 & $21,8 \%$ (894) \\
\hline HLA-DR8 & $11,47 \%(7)$ & $10,53 \%(41)$ & 0,826 & $11,9 \%(489)$ \\
\hline HLA-DR9 & $1,63 \%(1)$ & $2,31 \%(9)$ & 0,740 & $4,2 \%$ (174) \\
\hline HLA-DR10 & $1,63 \%(1)$ & $5,14 \%(20)$ & 0,228 & $4,5 \%(186)$ \\
\hline HLA-DR11 & $27,86 \%$ (17) & $26,47 \%(103)$ & 0,819 & $22,8 \%(933)$ \\
\hline HLA-DR12 & $1,63 \%(1)$ & $4,88 \%$ (19) & 0,253 & $3,7 \%(152)$ \\
\hline HLA-DR13 & $32,78 \%(20)$ & $27 \%(105)$ & 0,348 & $24,9 \%$ (1019) \\
\hline HLA-DR14 & $11,47 \%(7)$ & $9,76 \%(38)$ & 0,680 & $7,5 \%$ (306) \\
\hline HLA-DR15 & $16,39 \%(10)$ & $17,73 \%$ (69) & 0,797 & $21,1 \%(866)$ \\
\hline HLA-DR16 & $9,83 \%(6)$ & $4,62 \%(18)$ & 0,092 & $6,6 \%(271)$ \\
\hline
\end{tabular}


O tipo de doador também não foi preditor de DMPT, ${ }^{5}$ apesar de ser um dado controverso na literatura, pois alguns estudos não encontraram essa associação, no entanto o transplante de doador falecido já foi apontado como fator de risco para DMPT. Supostamente, inferimos que isto se deu pela diferença de imunossupressão quando comparado ao transplante renal com doador vivo. ${ }^{4,13,19}$ Apesar de $80 \%$ dos nossos pacientes serem portadores de hipertensão arterial sistêmica (HAS), não encontramos relação com risco de DMPT, ${ }^{5}$ embora outros estudos relatem essa associação. ${ }^{4}$

Apesar do grupo com DMPT ter utilizado dose cumulativa de corticosteróides, ligeiramente maior que o grupo sem DMPT, isso não influenciou no desenvolvimento de DMPT. ${ }^{5}$ Os corticosteróides apresentam efeito diabetogênico através de vários mecanismos, como piora da resistência insulínica, aumento da gliconeogênese hepática, aumento de peso e dislipidemia induzida por lipólise, além da redução da massa muscular, diminuição da captação de glicose e síntese de glicogênio nas células musculares esqueléticas. ${ }^{20,21}$ Outros autores relataram que a retirada de $5 \mathrm{mg}$ de prednisolona não altera sensibilidade à insulina de forma importante, podendo levar ao aumento do risco de rejeição aguda, piora da proteinúria e recorrência de glomerulonefrite, não sendo recomendada deste modo sua retirada do regime imunossupressor. ${ }^{22-24}$

Nosso estudo também não conseguiu identificar como fator de risco para DMPT o uso de nenhum imunossupressor incluído no regime terapêutico, ${ }^{5}$ conforme também discutido na literatura. ${ }^{4,25,26}$ No entanto, ressaltamos que o FK pode induzir a apoptose de células beta pancreáticas, diminuindo a exocitose de insulina, e reduzir a transcrição do gene da insulina, o que poderia contribuir para o desenvolvimento de DMPT. ${ }^{27}$

O Sirolimo pode levar ao aumento da gliconeogênese hepática e redução da captação de glicose induzindo DM por severa intolerância à glicose, hiperinsulinemia e hipertrigliceridemia. ${ }^{28-30} \mathrm{O}$ everolimo por sua vez apresenta menor impacto na função das células beta pancreáticas quando comparado ao sirolimo. ${ }^{27}$ Diante disso, pacientes que trocaram CsA por everolimo tiveram ocorrência de DMPT significativamente menor comparados aos que permaneceram em uso de CsA, além de melhor função renal, embora tenha causado taxas de rejeição mais altas do enxerto renal. ${ }^{31}$ Por outro lado, outro estudo não mostrou diferença na incidência ou gravidade da DMPT com conversão precoce de CsA para everolimo. ${ }^{32}$ Diante disso, também não encontramos relação entre o uso de imTOR e DMPT, ${ }^{5}$ possivelmente pelo baixo número de pacientes que utilizou essas drogas.

Além do HLA, outros fatores genéticos de risco ao DMPT têm sido relatados, como o CY2224 rs2296241, que foi associado ao risco de DMPT após pesquisa da associação de variáveis clínicas e cinco polimorfismos de nucleotídeo único (SNPs) selecionados com DMPT em receptores chineses de transplante renal em uso de tacrolimo ${ }^{33} \mathrm{~A}$ relação entre DMPT e biomarcadores relacionados à inflamação (fator de necrose tumoral solúvel tipo 1 , Pentraxin 3 , fator de inibição de migração de macrófagos e receptor endotelial de Proteína C) também foi evidenciada. ${ }^{34}$ Adicionalmente, uma variante genética no gene CYP4F2, o principal gene implicado na síntese de hidroxieicosatetraenóico, o qual desempenha um papel importante no transplante renal e DM, esteve associada ao risco de DMPT. ${ }^{35}$ Esses achados trazem perspectivas para que estudos futuros possam comprovar uma susceptibilidade genética para DMPT, oferecendo gradativamente base sólida para ações preventivas cada vez mais precoces, de modo a evitar seu desenvolvimento e, consequentemente, suas complicações para o paciente e para o enxerto renal.

Por fim, a prevenção e o tratamento do DMPT continuam sendo baseados na atuação sobre fatores de risco modificáveis, uma vez que fatores de risco não modificáveis ainda permanecem controversos. Mudança no estilo de vida, controle glicêmico rígido, introdução precoce de bloqueadores do sistema renina-angiotensina-aldosterona e escolha e ajuste do regime imunossupressor têm sido mostrados como ações fundamentais na redução de DMPT. ${ }^{36}$ Não menos importante que tais medidas é o tratamento farmacológico precoce do DMPT, tendo como opções terapêuticas disponíveis a metformina, meglitinidas, agonistas do glucagon-like peptide (GLP1), inibidores da dipeptidil dipeptidase (DPP4), inibidores do cotransportador sódio-glicose 2 (SGLT2) e insulina. ${ }^{37-40}$ Prevenção, identificação de pacientes sob risco de desenvolver DMPT e tratamento precoces não apenas reduzem a ocorrência de DMPT, mas também seu risco cardiovascular, uma vez que a doença cardiovascular é a maior causa de morte em receptores de transplante renal, além de ser responsável por $30 \%$ da perda do enxerto. ${ }^{41,42}$

Por ser um estudo retrospectivo tivemos limitações quanto aos dados faltantes nos prontuários, como proteinúria, ausência de teste oral de tolerância a glicose e hemoglobina glicada, além de insulina sérica e índices para avaliação de resistência insulínica, uma vez que não estão incluídos na rotina do serviço. 


\section{CONCLUSÃO}

Concluímos que a detecção precoce dos fatores de risco é importante pois pode ser útil para estratificação de risco dos pacientes para determinarmos estratégias adequadas para redução de ocorrência de DMPT.
O estudo de associação entre antígenos HLA e DMPT mostrou associação positiva com HLA- A23 e HLA-A29 e associação negativa com HLA-A68, porém novos estudos são necessários para confirmar esses achados.

\section{ABSTRACT}

Introduction: Post-transplant diabetes mellitus (PTDM) increases both morbidity and mortality, resulting in cardiovascular complications in kidney transplanted recipients, and loss of renal graft. Risk factors for PTDM are divided into modifiable and non-modifiable. The impact of human leucocyte antigens (HLA) on PTDM is a subject of debate in the literature. Purpose: To verify prevalence of PTDM and its relationship with genetic factors. Methods: 450 patients who have undergone renal transplantation were investigated as to the association between HLA-A, -B and DR antigens and PTDM. The frequencies of HLA-A, -B and -DR antigens were compared between recipients with and without PTDM diagnosis over three years after transplantation. Results: In the studied population, $60 \%$ were male, $47.2 \%$ were black and $57.8 \%$ received kidney from a deceased donor. PTDM was diagnosed in 61 patients (13.5\%), 315 $(70 \%)$ remained with normal blood glucose levels, and 74 (16.5\%) developed impaired fasting blood glucose. Positive PTDM associations were observed in relation to HLA-A23 (18\% vs $8.7 \%, P=0.024)$ and HLA-A29 $(13.1 \%$ vs $5.1 \%$, $P=0.017)$, and negative association with HLA- A68 (4.9\% vs $14.1 \%, P=0.046)$. Conclusion: We found a statistical relationship between PTDM and HLA-A23, HLA-A29 and HLA-A68. However, further studies are needed to reach a final conclusion whether genetic susceptibility factors are associated with the development of PTDM.

Descritores: Diabetes Mellitus; Kidney Transplantation; Risk Factors; HLA Antigens.

\section{Agradecimentos}

Os autores agradecem à CAPES (Coordenação de Aperfeiçoamento de Pessoal de Nível Superior) pela bolsa de Mestrado à Débora Dias de Lucena.

\section{REFERÊNCIAS}

1. Tufton N, Ahmad S, Rolfe C,Rajkariar R, Byrne C, Chowdhury TA. New-onset diabetes after renal transplantation. Diabet Med. 2014;31(11):1284-92.

2. Cosio FG, Kudva Y, Van der Velde M, Larson TS, Textor SC, Griffin MD, et al. New onset hyperglycemia and diabetes are associated with increased cardiovascular risk after kidney transplantation. Kidney Int. 2005;67(6):2415-21.

3. Kasiske BL, Snyder JJ, Gilbertson D, Matas AJ. Diabetes mellitus after kidney transplantation in the United States. Am J Transplant. 2003; 3(2):178-85.
4. Bastos Jr. MAV, Oliveira MS, Castro SH, Cunha EF, Moraes ERS, Ruzzani F, et al. Fatores de risco para o desenvolvimento de diabetes mellitus pós-transplante renal. Arq Bras Endocrinol Metab. 2005;49(2):271-7.

5. Lucena DD, de Sá JR, Medina-Pestana JO and Rangel EB. Modifiable Variables Are Major Risk Factors for Posttransplant Diabetes Mellitus in a Time-Dependent Manner in Kidney Transplant: An Observational Cohort Study. Journal of Diabetes Research. 2020; Volume 2020, Article ID 1938703, 10 pages. 
6. Torres-Romero LF, Santiago-Delpin EA, de Echegaray S,Solis DR, Rodriguez-Trinidad AT, Gonzalez-Caraballo ZA, et al. HLA is not predictive of posttransplant diabetes mellitus. Transplant Proc. 2006;38(3):914-5.

7. Nafar M, Pour-Reza-Gholi F, Amouzegar A, Einollahi B, Firouzan A, Hemati K,et al. Is HLA-DR6 a protective factor against posttransplantation diabetes mellitus?. Transplant Proc. 2005; 37(7):3098-100.

8. Yu SJ, Peng L, Xie XB, Peng FH, Fang $\mathrm{CH}$, Wang $\mathrm{Y}$, et al. Correlation Between HLA and Posttransplantation Diabetes Mellitus in the Han Population in South China. Transplant Proc. 2010; 42(7): 2509-12.

9. Sumrani NB, Delaney V, Ding ZK, et al. Diabetes mellitus after renal transplantation in the cyclosporine era--an analysis of risk factors. Transplantation. 1991;51(2):343-7. DOI: 10.1097/00007890-199102000-00014.

10.Nieuwenhuis MG, Kirkels JH. Predictability and other aspects of post-transplant diabetes mellitus in heart transplant recipients. J Heart Lung Transplant. 2001; 20(7):703-8. doi: 10.1016/s1053-2498(01)00257-1. PMID: 11448794.

11. Pietrzak-Nowacka M., Safranow K., Nowosiad M., DębskaŚlizień A., Dziewanowski K., Głyda M., Jankowska M., Rutkowski B. and Ciechanowski K. HLA-B27 is a Potential Risk Factor for Posttransplantation Diabetes Mellitus in Autosomal Dominant Polycystic Kidney Disease Patients. Transplantation Proceedings. 2010;42(9):3465-70.

12. Sharif A, Moore R, Baboolal K. Influence of Lifestyle Modification in Renal Transplant Recipients with Postprandial Hyperglycemia. Transplantation. 2008;85(3):353-8.

13. Alshamsi, S. Risk factors and their relative strengths for new onset diabetes after transplantation (nodat). Nephrol Dial Transplant. 2015;30(3):iii653.

14. Joss N, Staatz CE, Thomson AH, Jardine AG. Predictors of new onset diabetes after renal transplantation. Clin Transplant. 2007;21(1):136-43.

15. Campos EF, Doxiadis IIN, Temin J, Plothow A, Bellintani EC, Miyamoto $Y$, et al. Proposal for a program to enhance renal transplantation opportunity for highly sensitized patients. J Bras Transp. 2010; 13:1221-80

16. Porrini EL, Díaz JM, Moreso F, Delgado Mallén PI, Silva Torres I, Ibernon M, et al. Clinical evolution of post-transplant diabetes mellitus. Nephrol Dial Transplant. 2016;31(3):495505.

17. Teixeira APSF, Fernandes NMS, da Mata GF,Chaoubah A, de Paula RB, Bastos MG.Prevalência de síndrome metabólica e fatores associados em pacientes transplantados renais. J BrasNefrol 2012;34(1):16-21.

18. Baron PW, Infante S, Peters R, Tilahun J, Weissman J, Delgado L, et al. Post-Transplant Diabetes Mellitus After Kidney Transplant in Hispanics and Caucasians Treated with Tacrolimus-Based Immunosuppression. Am J Transplant. 2003;3(2):178-85.

19. Copstein L, Garcia JP, Zelmanovitz T, Gonçalves LF, Manfro RC. Post Transplant Diabetes Mellitus in Cyclosporine Treated Renal Transplant Recipients. Braz.J. Bras. Nefrol. 2008;30(1):59-65.
20. Kahn SE. The relative contributions of insulin resistance and beta-cell dysfunction to the pathophysiology of type 2 diabetes. Diabetologia2003;46:3-19.

21. Rangel EB. The metabolic and toxicological considerations for immunosuppressive drugs used during pancreas transplantation. Expert Opin Drug MetabToxicol. 2012;8(12):1531-48.

22. Midtvedt K,Hjelmesaeth J, Hartmann A,Lund K, Paulsen D, Egeland $T$, et al.Insulin resistance after renal transplantation: the effect of steroid dose reduction and withdrawal. J Am Soc Nephrol. 2004;15(12):3233-9.

23. Pascual J, Quereda C, Zamora J, Hernández D; Spanish Group for Evidence-Based Medicine in Renal Transplantation.Steroid withdrawal in renal transplant patients on triple therapy with a calcineurin inhibitor and mycophenolate mofetil: a meta-analysis of randomized, controlled trials. Transplantation. 2004;78:1548-56.

24. Leeaphorn N, Garg N, Khankin EV,Cardarelli F, Pavlakis M. Recurrence of IgA nephropathy after kidney transplantation in steroid continuation versus early steroidwithdrawal regimens: a retrospective analysis of the UNOS/OPTN database. Transpl Int. 2018;31:175-86.

25. Tillmann FP, Rump LC, Quack I. HbA1c levels at 90 days after renal transplantation in non-diabetic recipients predict de novo pre-diabetes and diabetes at 1 and 3 years after transplantation. Int UroINephrol. 2018;50(8):1529-34.

26. Cosio FG, Pesavento TE, Osei K, Henry ML, Ferguson RM. Post-transplant diabetes mellitus: increasing incidence in renal allograft recipients transplanted in recent years. Kidney Int. 2001;59(2):732-7.

27. Rangel, EB. Tacrolimus in pancreas transplant: a focus on toxicity, diabetogenic effect and drug-drug interactions. Expert OpinDrugMetabToxicol. 2014;10(11):1585-605.

28. Silva GF, de Paula MI, Rangel EB. mTOR inhibitors in pancreas transplant: adverse effects and drugdrug interactions. Expert Opin Drug MetabToxicol. 2017;13(4):367-385.

29. Morrisett JD, Abdel-Fattah G, Hoogeveen R, Mitchell E, Ballantyne CM, Pownall $\mathrm{HJ}$, et al. Effects of sirolimus on plasma lipids, lipoprotein levels, and fatty acid metabolism in renal transplant patients. J Lipid Res. 2002; 43:1170-80.

30. Teutonico A, Schena PF, Di PS. Glucose metabolism in renal transplant recipients: effect of calcineurin inhibitor withdrawal and conversion to sirolimus. J Am Soc Nephrol. 2005;16:3128-35.

31.Kälble F, Seckinge J, Schaier M, Morath C, Schwenger $\mathrm{V}$, Zeier M, et al. Switch to an everolimus-facilitated cyclosporine A sparing immunosuppression improves glycemic control in selected kidney transplant recipients. Clin Transplant. 2017;31(8):13067.

32.Sommerer C, Witzke O, Lehner F, Arns W, ReinkeP, Eisenberger $U$, et al. Onset and progression of diabetes in kidney transplant patients receiving everolimus or cyclosporine therapy: an analysis of two randomized, multicenter trials. BMC Nephrol. 2018 Sep 19;19(1):237. 
33.Zhang X, Men T, Liu H, Li X, Cai M. Genetic risk factors for post-transplantation diabetes mellitus in Chinese Han renal allograft recipients treated with tacrolimus. Transpl Immunol. 2018;49:39-42.

34. Heldal TF, Ueland T, Jenssen T, Hartmann A, Reisaeter $A V$, Aukrust $P$, et al. Inflammatory and related biomarkers are associated with post-transplant diabetes mellitus in kidney recipients: a retrospective study. Transpl Int. 2018;31(5):510-19.

35.Gervasini G, Luna E, García-Cerrada M,García-Pino G, Cubero JJ. Risk factors for post-transplant diabetes mellitus in renal transplant: Role of genetic variability in the CYP450-mediated arachidonic acid metabolism. Molecular and Cellular Endocrinology. 2016; 419: 15864.

36.KDIGO Clinical Practice Guideline for the Care of Kidney Transplant Recipient. Chapter 15: Diabetes Mellitus. American Journal of Transplantation 2009; 9 (Suppl 3): S65-S65.
37. Hornum M, Lindahl JP, Zur-Muhlen B, Jenssen T, FeldtRasmussen B. Diagnosis, management and treatment of glucometabolic disorders emerging after kidney transplantation. Transplant International. 2013; 26:1049-60.

38. Sharif A. Should metformin be our antiglycemic agent of choice post-transplantation? Am J Transplant. 2011;11(7):7681.

39. Larsen JL. Potential risks of metformin in transplant patients. Am J Transplant. 2012;12(3):795; author reply 796.

40. Hecking M, Haidinger M, Döller D, Werzowa J, Tura A, Zhang J,et al. Early basal insulin therapy decreases newonset diabetes after renal transplantation. J Am Soc Nephrol. 2012;23(4):739-49.

41. Hjelmesaeth J, Hartmann A, Midtvedt K, Aakhus S, Stenstrøm J, Mørkrid L,et al. Metabolic cardiovascular syndrome after renal transplantation. Nephrol Dial Transplant. 2001;16(5):1047-52.

42. Vella J, Lentine KL. Risk factors for cardiovascular disease in the renal transplant recipient. UpToDate 2014. 